\title{
Tissue localization during resective epilepsy surgery
}

\author{
Sandra L. Poliachik, Ph.D., ${ }^{1,2,6}$ Andrew V. Poliakov, Ph.D., ${ }^{2,3}$ \\ Laura A. Jansen, M.D., Ph.D., ${ }^{4,7}$ Sharon S. McDaniel, M.D., ${ }^{1,4}$ Carter D. Wray, M.D., ${ }^{1,4}$ \\ John Kuratani, M.D., ${ }^{1,4,6}$ Russell P. Saneto, D.O., Ph.D., $1,4,6$ \\ Jeffrey G. OJemann, M.D., ${ }^{3,5,7,8}$ AND EdWard J. Novotny JR., M.D., $1,4,8$
}

Divisions of ${ }^{1}$ Pediatric Neurology, ${ }^{2}$ Pediatric Radiology, and ${ }^{3}$ Pediatric Neurosurgery, Seattle Children's Hospital; Departments of ${ }^{4}$ Neurology and ${ }^{5}$ Neurosurgery, and ${ }^{8}$ Integrative Brain Imaging Center, University of Washington; and Centers for ${ }^{6}$ Clinical and Translational Research and ${ }^{7}$ Integrative Brain Research, Seattle Children's Research Institute, Seattle, Washington

\begin{abstract}
Object. Imaging-guided surgery (IGS) systems are widely used in neurosurgical practice. During epilepsy surgery, the authors routinely use IGS landmarks to localize intracranial electrodes and/or specific brain regions. The authors have developed a technique to coregister these landmarks with pre- and postoperative scans and the Montreal Neurological Institute (MNI) standard space brain MRI to allow 1) localization and identification of tissue anatomy; and 2) identification of Brodmann areas (BAs) of the tissue resected during epilepsy surgery. Tracking tissue in this fashion allows for better correlation of patient outcome to clinical factors, functional neuroimaging findings, and pathological characteristics and molecular studies of resected tissue.

Methods. Tissue samples were collected in 21 patients. Coordinates from intraoperative tissue localization were downloaded from the IGS system and transformed into patient space, as defined by preoperative high-resolution T1-weighted MRI volume. Tissue landmarks in patient space were then transformed into MNI standard space for identification of the BAs of the tissue samples.

Results. Anatomical locations of resected tissue were identified from the intraoperative resection landmarks. The BAs were identified for 17 of the 21 patients. The remaining patients had abnormal brain anatomy that could not be meaningfully coregistered with the MNI standard brain without causing extensive distortion.

Conclusions. This coregistration and landmark tracking technique allows localization of tissue that is resected from patients with epilepsy and identification of the BAs for each resected region. The ability to perform tissue localization allows investigators to relate preoperative, intraoperative, and postoperative functional and anatomical brain imaging to better understand patient outcomes, improve patient safety, and aid in research.
\end{abstract}

(http://thejns.org/doi/abs/10.3171/2013.3.FOCUS1360)

KEY WoRDS $\quad$ epilepsy $\bullet \quad$ neuroimaging modality $\bullet \quad$ imaging-guided surgery $\bullet$
image coregistration $\bullet$ resective epilepsy surgery

$\mathrm{M}$ ORE than 50 million people worldwide have epilepsy. Approximately $25 \%-35 \%$ of these individuals do not respond to antiepileptic medication or dietary interventions and should be evaluated for possible epilepsy surgery. Imaging is an integral part of the comprehensive preoperative evaluation for epilepsy, supporting identification of a specific part of the brain as the epileptic focus (for example, structural or metabolic abnormality). Imaging-guided surgery systems are now widely used in surgical practice, especially in neurosurgery. ${ }^{4,15}$ Navigation systems do not automatically link

Abbreviations used in this paper: $\mathrm{BA}=$ Brodmann area; $\mathrm{EEG}$ = electroencephalography; fcMRI = functional connectivity MRI; fMRI = functional MRI; IGS = imaging-guided surgery; MNI = Montreal Neurological Institute. pre- and postoperative imaging data, nor do they allow for localizing resected tissue with respect to the imaging data. Particularly in epilepsy surgery, and when tissue is being used for both diagnostic and research purposes, it is important to be able to relate preoperative and postoperative imaging data with intraoperative tissue localization. Integrating the intraoperative data from the IGS with imaging could not only improve surgical accuracy and thus patient outcomes and safety, but can advance research as well.

Neuroimaging techniques used in the preoperative epilepsy evaluation include structural MRI and fMRI, metabolic evaluations based on PET imaging, and the assessment of blood flow in the brain during ictal and/or interictal activity by using SPECT scanning. Scalp electrodes used in EEG recordings provide general localiza- 
tion for seizure activity and can be further enhanced by combining with imaging for electrical source imaging. Also, magnetoencephalography is combined with imaging in magnetic source imaging. Often, if the results are concordant, these studies may identify a specific region suitable for resection. However, some patients may require stereo-EEG or intracranial EEG to tailor a resection and minimize morbidity. If an intracranial EEG study is undertaken based on these findings, CT structural imaging may be used to identify the intracranial electrode locations.

As an essential part of any neurosurgical planning and navigation system, image registration facilitates combining images with complementary structural and functional information to improve the information on which a surgeon makes critical decisions. Image registration is the process of transforming images acquired at different time points, from different imaging systems, or with different imaging modalities into the same coordinate system. This procedure is an integral part of neurosurgery for brain tumors, movement disorders, and epilepsy.

Intraoperative tools used during intracranial EEG studies and resections are available to assist the neurosurgeon in electrode localization or tissue identification. Image-guided surgery systems can coregister preoperative imaging data from structural MRI to fMRI, PET, and SPECT studies that are overlaid onto anatomical MRI scans, allowing the neurosurgeon to identify relevant tissues during procedures. In addition, most IGS systems allow the neurosurgeon to perform virtual labeling of anatomical regions or electrodes by using landmarks in a coordinate system that is specific to the IGS system.

We have developed a technique in a pediatric population in which we used intraoperative surgical landmarks to assist in identifying intracranial EEG electrode placement and in localizing the tissue removed during resective epilepsy surgery. Data from intracranial EEG studies and resective epilepsy surgeries (landmarks generated by the IGS system) are coregistered with pre- and postoperative structural, functional, and metabolic neuroimaging as well as postoperative CT data (used to build a model of implanted electrodes) by using BioImage Suite. ${ }^{16}$ These landmarks are used to verify image coregistration; during resective epilepsy surgery, additional landmarks are useful to track the origin of tissue samples that can be analyzed for gene and protein expression as well as pathological characteristics. The tissue landmarks are then transformed into standard MRI space (for example, MNI or Talairach) to identify the standard structural regions (for example, BAs) of the tissue specimens resected during epilepsy surgeries. This report describes using multimodal image coregistration coupled with IGS landmarks and resection to verify the anatomy of resected tissue in 21 patients.

\section{Methods}

\section{Patient Population}

Patients included in the study presented with seizures that became medically intractable. Each patient under- went resective epilepsy surgery during which at least 1 tissue sample was tracked with an IGS landmark. Imaging for patients included at least 2 of the following scans: structural MRI, anesthetized or awake fMRI (passive sensory motor task, resting state), PET, and/or SPECT. For each patient, relevant studies were downloaded onto a Medtronic (StealthStation TREON) IGS system for coregistration with the patient's presurgical MRI that included fiducial markers.

\section{Neuroimaging Examinations}

All MRI examinations were completed on a Siemens 3T (MAGNETOM Trio) unit equipped with a 32-channel head coil. Retrospective institutional review board approval was obtained by Seattle Children's Hospital for inclusion of all participants in this report.

Structural MRI. The standard clinical epilepsy imaging protocol included a high-resolution sagittal T1weighted magnetization-prepared rapid gradient echo, axial T2-weighted, axial FLAIR, and coronal T2-weighted scans. In addition, diffusion tensor sequences were collected with the following parameters: TE $86 \mathrm{msec}, \mathrm{b}$ $1000 \mathrm{sec} / \mathrm{mm}^{2}$, 30 directions repeated 2-4 times, $2 \times 2$ $\mathrm{mm}$ in-plane resolution, 2.2-mm slice thickness, 55-60 slices.

Functional MRI. The fMRI data were collected at Seattle Children's Hospital according to clinical protocols for language and motor mapping. ${ }^{17}$ The fMRI was performed using an echo planar imaging blood oxygen level-dependent sequence (Siemens clinical sequence, ep2d PACE [Prospective Acquisition Correction]), with the following parameters: 45 slices, slice thickness $3 \mathrm{~mm}$, TE $30 \mathrm{msec}$, TR 2400-3000 msec, flip angle 90 , FOV $192 \times 192 \mathrm{~mm}$, matrix $64 \times 64$. Eighty volumes were obtained per run, resulting in a total time of approximately 4 minutes per scan. For the patients in Cases 1 and 3, the study was designed in an ABABABAB algorithm in which the 2 tasks, A and B, were performed for 10 volumes each. Each patient was instructed to tap his or her fingers. In children too young to cooperate, somatosensory cortex was activated by passive movement with the patient under sedation. The verb generation task alternated between fixation point control and the specific language task that consisted of silent verb generation based on pictures or words. Postprocessing analysis was performed using the commercially available Siemen's software and FSL software.

Functional Connectivity MRI. The fcMRI was performed with the following protocol: echo planar imaging blood oxygen level-dependent sequence: TE $30 \mathrm{msec}$, flip angle $90^{\circ}$. The fcMRI scans were performed in both awake and anesthetized patients and were incorporated into either the clinical anatomical MRI or the tasked-based fMRI studies that were part of the patients' routine preoperative evaluation. Conscious patients were instructed to rest and remain relaxed with their eyes open, and sedated patients had the minimal required sedation (typically propofol) for movement control. Postprocessing of fcMRI data was performed using software developed by the Func- 
tional Connectomes Project (http://www.nitrc.org/projects/ fcon_1000/). Seed regions were defined based on previously published foci. ${ }^{9}$

Networks were identified post hoc by a seed-based analysis to identify sensorimotor, visual, auditory, and default mode networks by using MNI-based regions of interest. $^{2}$

Head CT Scans. In each patient who underwent intracranial EEG, head CT studies were obtained on a 64-slice multidetector CT unit (GE Lightspeed VCT, GE Healthcare). The imaging parameters used were $120 \mathrm{kV}$ and $300-335 \mathrm{~mA}$. Slice thickness was either 0.625 or 1.25 $\mathrm{mm}$, at a pixel size of $0.43 \times 0.43 \mathrm{~mm}$.

Fasting PET/CT Scans. Fasting PET scans were acquired using FDG on a GE Discovery STE scanner. Patients in whom PET scans were obtained underwent similar preparation (6-hour period with nothing by mouth except water; warm and quiescent uptake environment), scans were acquired 1 hour after injection of ${ }^{18} \mathrm{~F}-\mathrm{FDG}$, and images were obtained during quiet breathing (nonbreath-holding) for the PET and CT acquisition. ${ }^{1}$ To ensure that the PET was a truly interictal study, continuous EEG was performed during the entire procedure, both during the injection of the FDG and during image acquisition.

\section{Image Coregistration}

All patient space coregistrations used a high-resolution preoperative T1-weighted image as the base image to which all other scans were registered by using functions available in BioImage Suite. ${ }^{16}$ Skull stripping was accomplished using the brain extraction tool in FSL. ${ }^{18}$ Preoperative PET data, MRI FLAIR studies (when applicable), and postoperative CT scans obtained following grid implantation were coregistered to the preoperative T1-weighted image by using rigid linear registration based on normalized mutual information. In intracranial EEG studies, the postoperative CT data were used to build a model of the implanted electrodes with the BioImage Suite Electrode Editor program. Relevant task-based fMRI data in standard space were coregistered to the preoperative T1weighted base image by using a nonlinear registration. Overlays of the fMRI and PET results on the preoperative T1-weighted image were used to assess electrode location following implantation surgery. The preoperative T1-weighted image was coregistered to the 1-mm MNI standard space by using a nonlinear registration for identification of the resected tissue. When available, postoperative MRI data (high-resolution T1-weighted or FLAIR images) were linearly coregistered to the preoperative T1-weighted image to assess resection margins relative to EEG data.

\section{Intracranial Studies}

In those patients who were evaluated using intracranial EEG, monitoring continued for at least 3 days. Electrodes were either grids $(8 \times 8,7.5$ - or 10 -mm interelectrode spacing [Integra LifeSciences]); strips $(2 \times 8,1 \times$ $12,1 \times 8$, or $1 \times 4$ [Integra]); or depths (8 contact arrays with 5-mm spacing [Ad-Tech Corp.]) that were custommade to accommodate the AxiEm navigation system (Medtronic Corp.) as previously reported. ${ }^{20}$ Intracranial electrodes were placed based on information gathered during scalp ictal video-EEG and on neuroimaging data. During electrode implantation, tissue landmarks (surgical plans consisting of target and entry points) were collected and stored on the IGS system. The tissue landmarks were downloaded from the IGS system, and a landmark file was built in BioImage Suite. The landmarks were transformed from the IGS space to the patient space MRI (preoperative structural T1-weighted image) by using BioImage Suite.

\section{Resective Epilepsy Surgery/Brodmann Area Identification}

Tissue samples were collected in the patients undergoing resective epilepsy surgery. Landmark coordinates from intraoperative tissue localization were downloaded from the IGS system and transformed into patient space by using BioImage Suite. After tissue landmarks and patient space were transformed into MNI standard space, the Brodmann areas atlas tool in Biolmage Suite was used to identify the areas that were labeled intraoperatively with the IGS system.

\section{Results}

The 21 patients included in the study ranged in age from 1 to 18 years (12 female and 9 male patients), and presented with seizures that evolved into drug-resistant epilepsy based on International League Against Epilepsy criteria. ${ }^{10}$ Imaging for all patients included structural MRI, anesthetized or awake fMRI (passive sensory motor task, resting state), and some patients also had PET ( $\mathrm{n}=$ $15)$ and/or SPECT $(n=1)$ scans. Eight patients were evaluated by intracranial EEG monitoring prior to resective epilepsy surgery, and in 14 patients postoperative structural MRI scans were obtained. All surgeries occurred within a 2-year period at Seattle Children's Hospital. Four patients had abnormal brains that precluded them from BA identification due to inability to be meaningfully registered to the MNI brain image. Table 1 summarizes patients' imaging modality, intracranial EEG status, type of resective surgery, and identified BAs.

The ability to coregister the PET and T1-weighted MRI, or the fMRI task results with the T1-weighted MRI, was helpful in planning the resective epilepsy surgery and intracranial EEG study (if used). An example of PET-MRI T1-weighted image coregistration is presented in Fig. 1, showing PET-MRI overlay images and an example of the resected posterior parietal tissue landmark for the patient in Case 21. This patient also underwent an intracranial EEG study, with electrodes shown in Fig. 1B and $\mathrm{C}$. Figure 2 displays the intracranial EEG electrodes and fMRI results on 3D renderings of the patient in Case 10. In Fig. 2 left, the intracranial EEG electrodes are overlaid with fMRI results for a right finger tap task, which is useful in planning resections. Tissue in BA 8 was removed from the superior frontal gyrus, as indicated by the landmark on the postoperative MRI (Fig. 2 right). The pre- and postoperative T1-weighted MRIs for the patient 
TABLE 1: Data on resective surgery in 21 patients with medically intractable epilepsy*

\begin{tabular}{|c|c|c|c|c|c|}
\hline $\begin{array}{l}\text { Case } \\
\text { No. }\end{array}$ & $\begin{array}{l}\text { Age (yrs), } \\
\text { Sex }\end{array}$ & $\begin{array}{l}\text { Other } \\
\text { Imaging }\end{array}$ & $\begin{array}{l}\text { Intracranial } \\
\text { EEG }\end{array}$ & Tissue Resected & BAs \\
\hline 1 & $6, M$ & PET & yes & partial It temporal lobectomy & 38 \\
\hline 2 & $5, F$ & PET & no & It hemispherectomy & NA \\
\hline 3 & $3, M$ & PET & no & It temporal partial lobectomy, glioma, or cortical dysplasia & 20,21 \\
\hline 4 & $7, \mathrm{~F}$ & PET & no & rt partial hemispherectomy & NA \\
\hline 5 & $14, \mathrm{M}$ & & no & rt parietal tumor & 39 \\
\hline 6 & $18, \mathrm{M}$ & & yes & rt frontal cavernous malformation & 40 \\
\hline 7 & $5, \mathrm{~F}$ & PET & yes & hemispherectomy & NA \\
\hline 8 & $10, F$ & PET & no & hemispherectomy & $20,21,40,46$; insula \\
\hline 9 & $11, \mathrm{~F}$ & & no & It temporal lobe surgical defect; low-grade glioma & 9, 21, 38; hippocampus \\
\hline 10 & $13, M$ & PET & yes & It cingulate, It ant frontal lobe, multiple subpial transections & $\begin{array}{l}6,8,24 ; \text { corpus callosum; primary sen- } \\
\text { sory; white matter }\end{array}$ \\
\hline 11 & $16, \mathrm{~F}$ & PET & no & subtotal rt frontal lobectomy & $8,9,10 ;$ primary motor \\
\hline 12 & $1, M$ & PET & no & focal rt occipitoparietal cortical dysplasia & $19 ;$ visual association; fusiform \\
\hline 13 & $8, F$ & PET & yes & partial It temporal lobectomy & $\begin{array}{l}\text { 20, } 21,22,38 \text {; hippocampus; amygdala; } \\
\text { ant temporal lobe }\end{array}$ \\
\hline 14 & $6, F$ & PET & no & hemispherectomy & $\begin{array}{l}6,11,21,22,44 ; \text { parahippocampal } \\
\text { gyrus; amygdala; caudate }\end{array}$ \\
\hline 15 & $2, \mathrm{~F}$ & & no & It temporal tumor (rare seizures) & parahippocampal gyrus \\
\hline 16 & $18, F$ & & yes & It frontal lobe lesion & 6,8 \\
\hline 17 & $1, \mathrm{~F}$ & PET & no & $\begin{array}{l}\text { hemispherectomy; large portion of pst It frontal lobe, It tem- } \\
\text { poral lobe, \& It ant parietal lobe }\end{array}$ & NA \\
\hline 18 & $9, \mathrm{~F}$ & PET & no & partial It temporal lobectomy & $\begin{array}{l}\text { 20, 38; amygdala; hippocampus, ant } \\
\text { temporal lobe; pst hippocampus }\end{array}$ \\
\hline 19 & $9, \mathrm{M}$ & & no & It mesial temporal lobe lesion & $\begin{array}{l}\text { 21, } 22,38 \text {; head hippocampus; amyg- } \\
\text { dala }\end{array}$ \\
\hline 20 & $3, M$ & PET & yes & partial focal It parietal lobe in It supramarginal gyrus region & 40; primary sensory \\
\hline 21 & $9, \mathrm{M}$ & PET, SPECT & yes & rt parietooccipital lobectomy w/ pst frontal involvement & $7,19,39$; primary sensory \\
\hline
\end{tabular}

* All patients underwent MRI, fMRI, and fcMRI scans. Abbreviations: ant = anterior; NA = not available; pst = posterior.

in Case 9, displayed in Fig. 3, show a temporal lobe lesion in the preoperative image. The tissue resected from the hippocampus, also identified in MNI space as BA hippocampus, is highlighted and marked by crosshairs in both the pre- (left) and postoperative (right) images.

\section{Discussion}

These cases demonstrate the techniques used to identify and track tissue that is resected in patients with epilepsy for pathological analysis, and assessment of gene and protein expression. ${ }^{8}$ Data from EEG and neuroimaging studies are combined to plan the intracranial electrode placement and tissue resection. Tracking of electrode placement and subsequent localization of tissue resection allows for correlation of intracranial EEG data, verification of the anatomy of the resected tissue, and pathological investigation of the tissue samples.

Using neuroimaging data to localize specific brain structures has been pursued via several methods. Whereas some studies in ex vivo tissue used techniques such as needle landmarks to localize excised tissue, ${ }^{12}$ other methods include laser range scanner registration ${ }^{3}$ and micro- scope tracking via stereopsis for 3D cortical surface estimation. ${ }^{19}$ In addition, mathematical techniques such as linear elastic modeling ${ }^{5,21}$ or quantitative $\mathrm{MRI}^{7}$ have been used to assess tissue location with respect to imaging data. None of these studies, however, has developed methods specifically to identify tissue based on BAs in conjunction with neuroimaging data.

Electrodes localized through postoperative CT and IGS system landmarks allowed verification of electrode locations and thus provided a more accurate coregistration of tissue samples in those patients who underwent intracranial EEG studies. An earlier study tracked electrode locations by using postoperative MRI and CT data in conjunction with intraoperative stereotactic navigational probe coordinates to estimate the shift in electrode location. ${ }^{11}$ Although we could make estimates of electrode shifts, our main goal was to use tissue tracking in conjunction with the outcome data, allowing the epilepsy care team to evaluate the outcomes of the resection with specific knowledge of the neuroimaging localization and composition of the resected tissue. For example, in Fig. 1A tissue was resected from the right parietooccipital region in the patient in Case 21 and corresponded to MRI 

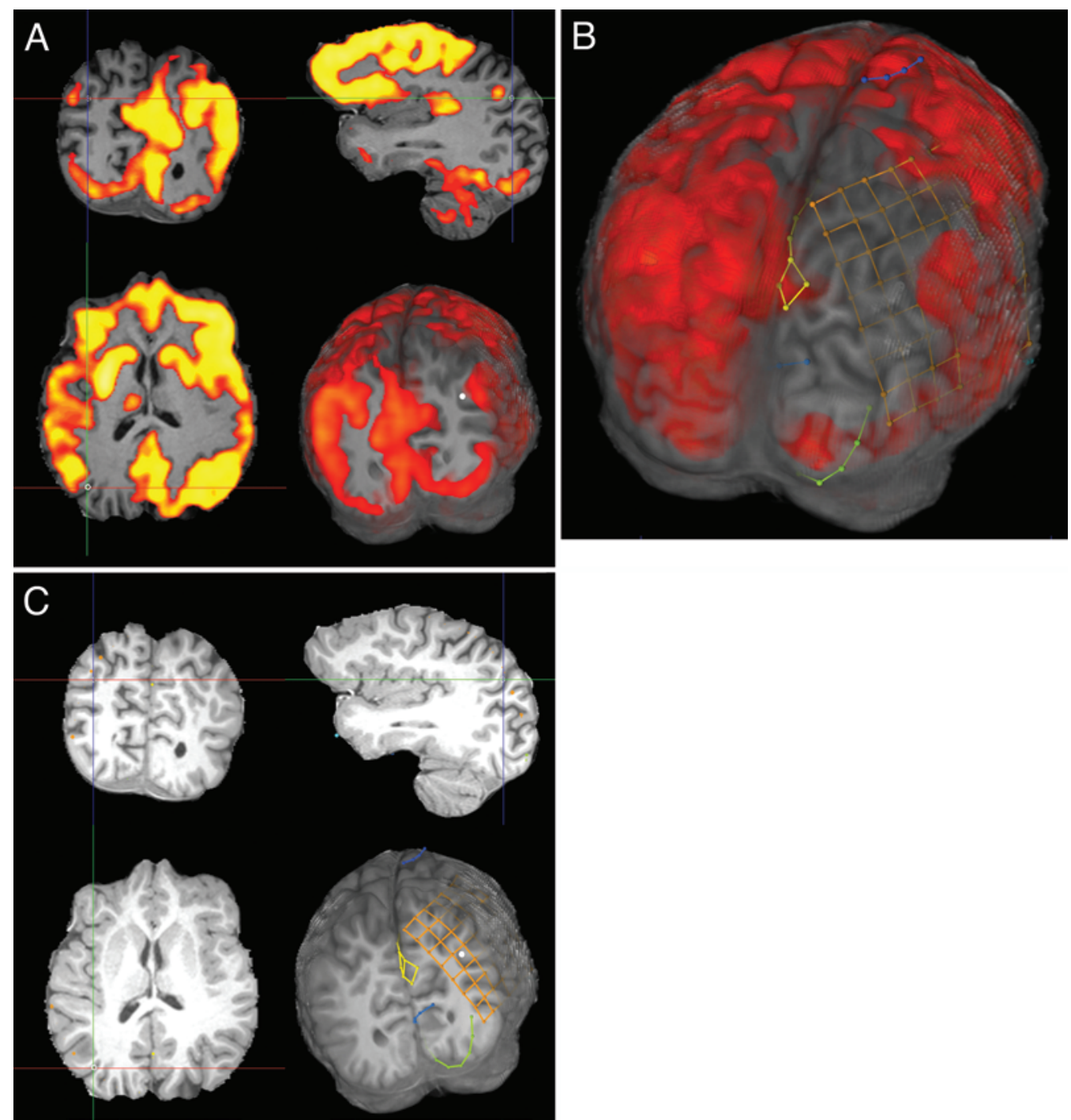

Fig. 1. Case 21. The landmark indicating the tissue that was resected from the posterior parietal region (BA 39) is displayed on preoperative images. The PET scan was thresholded to indicate metabolic activity in gray matter (high activity in yellow; low activity in red; hypometabolism is indicated by lack of a color overlay on gray matter). A: A PET scan coregistered to a T1-weighted MRI. The landmark for a posterior parietal tissue resection is indicated by the crosshairs on the 3-slice view and is displayed in white on the 3D view (trimmed to the depth of the landmark). B: A 3D rendering of a PET scan coregistered to a T1-weighted MRI, also showing intracranial EEG electrodes. C: A T1-weighted MRI showing tissue resected in BA 39. The landmark for BA 39 is indicated by crosshairs in the 3-slice view, and the intracranial EEG electrodes are overlaid on the 3D view, which is trimmed to show the landmark (displayed in white) indicating the posterior parietal tissue sample.

abnormalities identified with the help of PET. The BA for this tissue was identified as BA 39, angular gyrus, and is useful in predicting patient outcomes.

One of the main limitations of this tissue localization technique is the brain deformation that occurs intraoperatively as well as postoperatively. The difference in these locations was dependent on the size of the craniotomy, location of the landmark with respect to the craniotomy, position of the patient, and the extent and type of tissue resected, and is impacted by previous resective epilepsy surgeries. Several studies have attempted to estimate brain deformation and surgical changes that occur as a result of craniotomy for intracranial EEG and resective surgery. One such study used an algorithm incorporating tissue constraints, skull rigidity, and the fluidity of the
CSF in a 2D representation of the surgical site. ${ }^{6}$ Another method incorporated neuroimaging and game theory to account for brain surface changes. ${ }^{4}$ In some cases, intraoperative MRI allowed multiple intraoperative image acquisitions to evaluate brain shift. ${ }^{14}$ In addition, intraoperative MRI has been used to gather single-slice structural T1-weighted images to guide and localize the operation during conventional brain biopsy procedures. ${ }^{13}$

There are other limitations to this technique as well, including the quality and resolution of the imaging used in surgical planning and postoperative imaging. Although MRI data can be of sufficient resolution, PET data are generally of lower quality. During tissue resection, the IGS landmark is a point in space that is not indicative of the amount of tissue removed, and because that tis- 

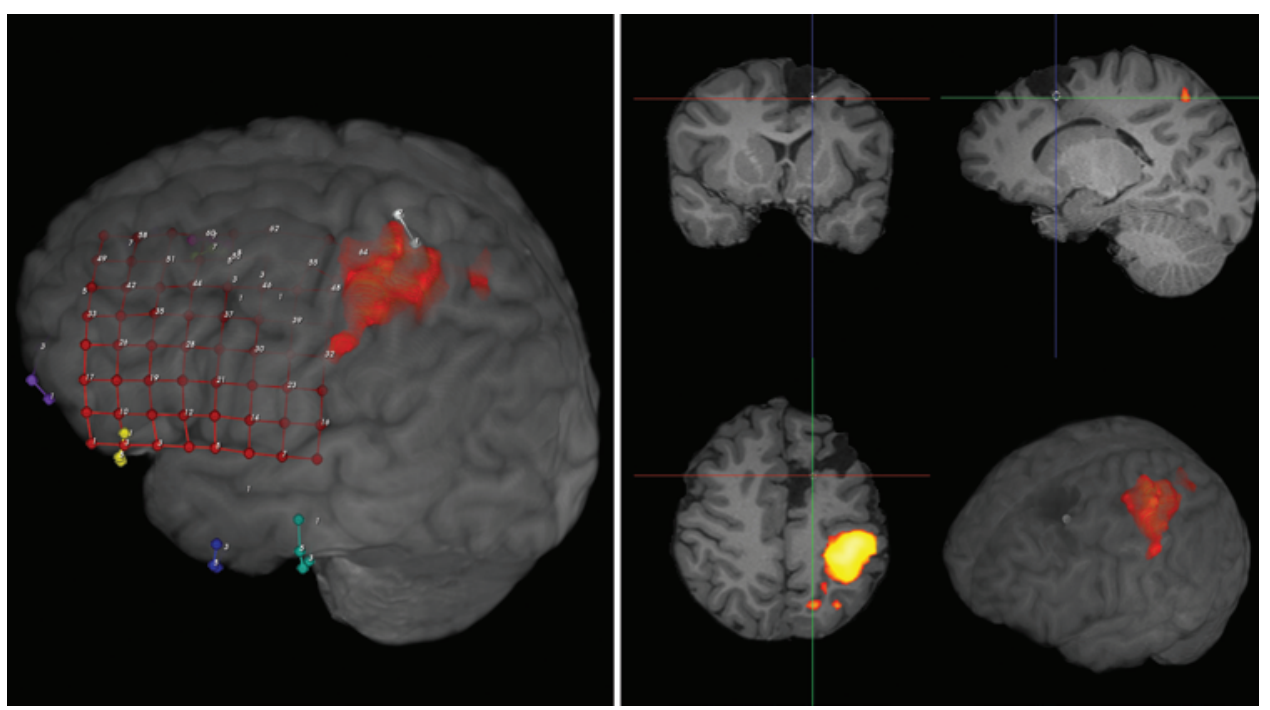

FIG. 2. Case 10. The $3 \mathrm{D}$ renderings obtained in this patient showing intracranial EEG electrode overlays on MR images. Left: The $f M R I$ for the right finger tap task and intracranial EEG electrodes are overlaid on the preoperative 3D T1weighted MRI volume. Right: The landmark indicating superior frontal tissue removed from BA 8 for this patient is shown in the postoperative 3-slice view and on the 3D volume. The landmark for tissue resected from BA 8 is displayed in white, indicated by the crosshairs in the 3-slice view.

sue is handled postresection, its orientation may not be adequately tracked. Also a challenge, as seen in the cases reported here, are previous resections or an unusual brain structure in which not only is brain deformation enhanced, but the abnormal anatomy of the brain may make tissue identification difficult when standard atlases are used.

Despite the limitations of this method, it is advantageous to have the ability to localize resected tissue. Some of the issues mentioned above are limited in an intact brain, and the tissue localization technique can be useful in identifying tissue anatomy, relating the resected tissue to the electrophysiological and imaging data, and tracking the resulting pathological and molecular analysis associated with each tissue sample. In addition, identification of the BAs in which tissue specimens were obtained can enhance the understanding of tissue composition.

\section{Conclusions}

This technique allows localization, anatomical identification, and subsequent tracking of tissue that is resected in patients with epilepsy. The identification of the BAs associated with each resected piece of tissue can typically
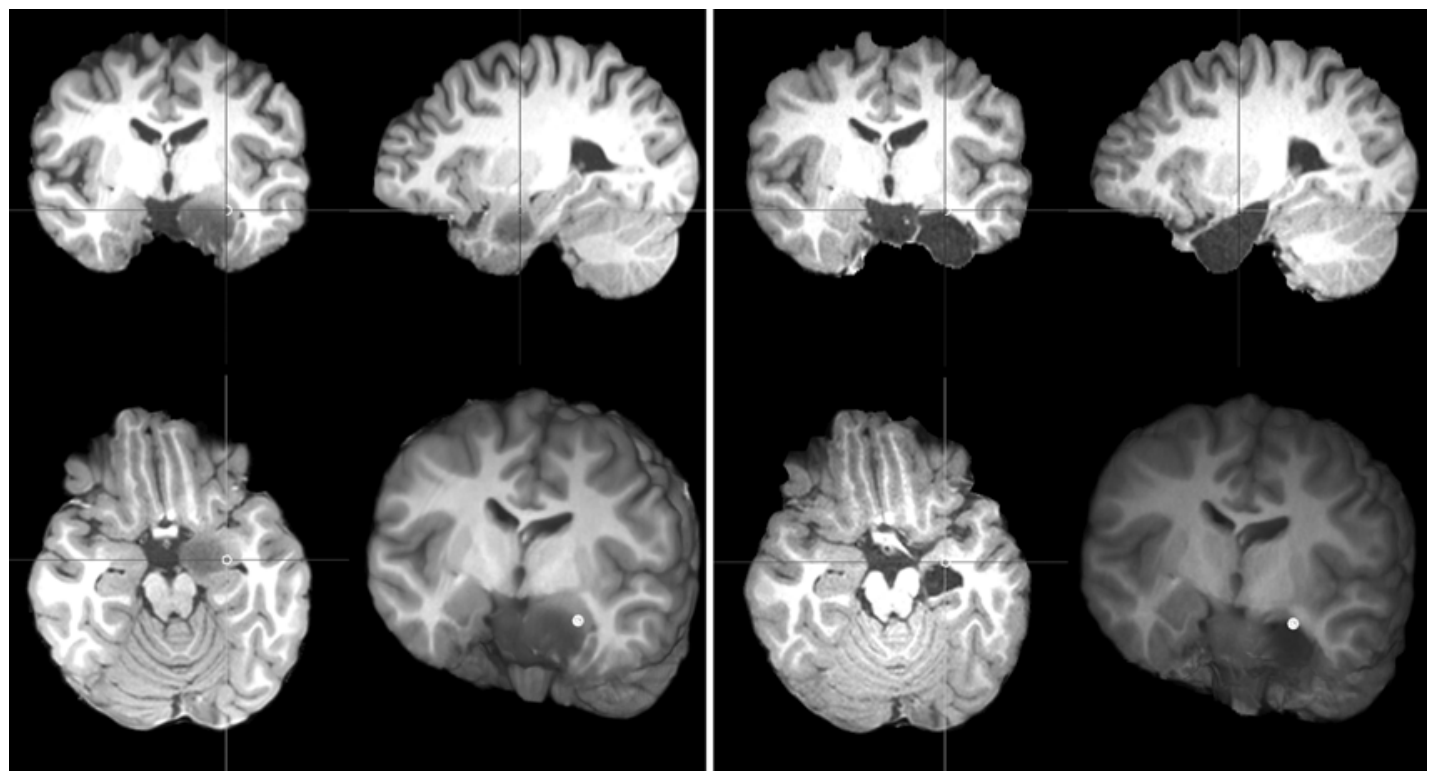

FIG. 3. Case 9. Pre- and postoperative T1-weighted MRI studies. Left: Preoperative T1-weighted MRI showing crosshairs over the landmark for tissue resected from the BA for hippocampus, near a temporal lobe lesion. The landmark is displayed in white on the 3D rendering of the MRI. Right: The landmark for the same BA shown on the left is displayed on the postoperative T1-weighted MRI. 
be accomplished, with an accuracy that is directly influenced by the amount of tissue deformation that occurs following surgery. Methods to compensate for the degree of deformation are available depending on the degree of accuracy required. ${ }^{4}$ Further processing of resected brain tissue for pathological characteristics and gene or protein expression can be correlated with clinical characteristics, clinical neurophysiology data, and functional neuroimaging ${ }^{17}$ studies on the same patient in an effort to improve patient outcomes.

\section{Disclosure}

The authors report no conflict of interest concerning the materials or methods used in this study or the findings specified in this paper.

Author contributions to the study and manuscript preparation include the following. Conception and design: Poliachik, Ojemann, Novotny. Acquisition of data: all authors. Analysis and interpretation of data: Poliachik, Jansen, McDaniel, Wray, Kuratani, Saneto, Ojemann, Novotny. Drafting the article: Poliachik, Jansen, Novotny. Critically revising the article: Poliachik, Poliakov, Jansen, Wray, Ojemann, Novotny. Reviewed submitted version of manuscript: Poliakov, McDaniel, Wray, Kuratani, Saneto, Ojemann, Novotny. Approved the final version of the manuscript on behalf of all authors: Poliachik. Administrative/technical/material support: Poliachik, Novotny. Study supervision: Ojemann, Novotny.

\section{References}

1. Alessio AM, Kinahan PE, Manchanda V, Ghioni V, Aldape L, Parisi MT: Weight-based, low-dose pediatric whole-body PET/CT protocols. J Nucl Med 50:1570-1577, 2009

2. Biswal B, Yetkin FZ, Haughton VM, Hyde JS: Functional connectivity in the motor cortex of resting human brain using echo-planar MRI. Magn Reson Med 34:537-541, 1995

3. Cao A, Thompson RC, Dumpuri P, Dawant BM, Galloway $\mathrm{RL}$, Ding S, et al: Laser range scanning for image-guided neurosurgery: investigation of image-to-physical space registrations. Med Phys 35:1593-1605, 2008

4. DeLorenzo C, Papademetris X, Staib LH, Vives KP, Spencer DD, Duncan JS: Image-guided intraoperative cortical deformation recovery using game theory: application to neocortical epilepsy surgery. IEEE Trans Med Imaging 29:322-338, 2010

5. DeLorenzo C, Papademetris X, Staib LH, Vives KP, Spencer DD, Duncan JS: Volumetric intraoperative brain deformation compensation: model development and phantom validation. IEEE Trans Med Imaging 31:1607-1619, 2012

6. Edwards PJ, Hill DL, Little JA, Hawkes DJ: A three-component deformation model for image-guided surgery. Med Image Anal 2:355-367, 1998

7. Eriksson SH, Free SL, Thom M, Martinian L, Symms MR, Salmenpera TM, et al: Correlation of quantitative MRI and neuropathology in epilepsy surgical resection specimens-T2 correlates with neuronal tissue in gray matter. Neuroimage 37:48-55, 2007

8. Jansen LA, Peugh LD, Roden WH, Ojemann JG: Impaired maturation of cortical GABA(A) receptor expression in pediatric epilepsy. Epilepsia 51:1456-1467, 2010
9. Johnston JM, Vaishnavi SN, Smyth MD, Zhang D, He BJ, Zempel JM, et al: Loss of resting interhemispheric functional connectivity after complete section of the corpus callosum. J Neurosci 28:6453-6458, 2008

10. Kwan P, Arzimanoglou A, Berg AT, Brodie MJ, Allen Hauser W, Mathern G, et al: Definition of drug resistant epilepsy: consensus proposal by the ad hoc Task Force of the ILAE Commission on Therapeutic Strategies. Epilepsia 51:1069-1077, 2010

11. LaViolette PS, Rand SD, Ellingson BM, Raghavan M, Lew SM, Schmainda KM, et al: 3D visualization of subdural electrode shift as measured at craniotomy reopening. Epilepsy Res 94: 102-109, 2011

12. Lazebnik RS, Lancaster TL, Breen MS, Lewin JS, Wilson DL: Volume registration using needle paths and point landmarks for evaluation of interventional MRI treatments. IEEE Trans Med Imaging 22:653-660, 2003

13. Moriarty TM, Quinones-Hinojosa A, Larson PS, Alexander E III, Gleason PL, Schwartz RB, et al: Frameless stereotactic neurosurgery using intraoperative magnetic resonance imaging: stereotactic brain biopsy. Neurosurgery 47:1138-1146, 2000

14. Nabavi A, Black PM, Gering DT, Westin CF, Mehta V, Pergolizzi RS Jr, et al: Serial intraoperative magnetic resonance imaging of brain shift. Neurosurgery 48:787-798, 2001

15. Papademetris X, DeLorenzo C, Flossmann S, Neff M, Vives KP, Spencer DD, et al: From medical image computing to computer-aided intervention: development of a research interface for image-guided navigation. Int J Med Robot 5:147-157, 2009

16. Papademetris X, Jackowski M, Rajeevan N, DiStasio M, Okuda H, Constable RT, et al: BioImage Suite: an integrated medical image analysis suite: an update. Insight J, 2006 (http:// hdl.handle.net/1926/209) [Accessed March 29, 2013]

17. Shurtleff H, Warner M, Poliakov A, Bournival B, Shaw DW, Ishak G, et al: Functional magnetic resonance imaging for presurgical evaluation of very young pediatric patients with epilepsy. Clinical article. J Neurosurg Pediatr 5:500-506, 2010

18. Smith SM: Fast robust automated brain extraction. Hum Brain Mapp 17:143-155, 2002

19. Sun H, Roberts DW, Farid H, Wu Z, Hartov A, Paulsen KD: Cortical surface tracking using a stereoscopic operating microscope. Neurosurgery 56 (1 Suppl):86-97, 2005

20. Wray CD, Kraemer DL, Yang T, Poliachik SL, Ko AL, Poliakov A, et al: Freehand placement of depth electrodes using electromagnetic frameless stereotactic guidance. Technical note. J Neurosurg Pediatr 8:464-467, 2011

21. Zhang C, Wang M, Song Z: A brain-deformation framework based on a linear elastic model and evaluation using clinical data. IEEE Trans Biomed Eng 58:191-199, 2011

Manuscript submitted February 14, 2013.

Accepted March 8, 2013.

Portions of this work were presented in abstract and poster form at the American Epilepsy Society Annual Meeting in Baltimore, Maryland, on December 4, 2011.

Please include this information when citing this paper: DOI: 10.3171/2013.3.FOCUS1360.

Address correspondence to: Sandra Poliachik, Ph.D., 4800 Sand Point Way, MS MA.7.220, Seattle, Washington 98105. email: sandy.poliachik@seattlechildrens.org. 